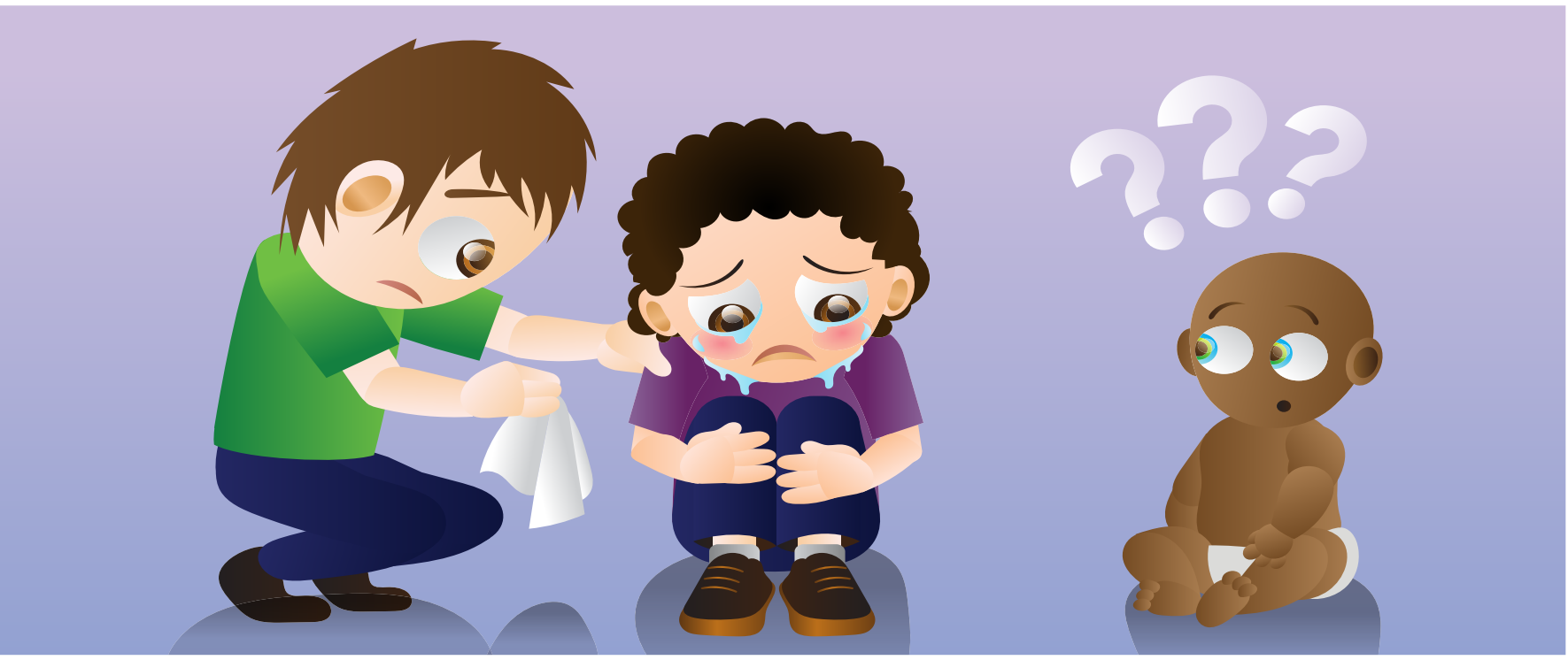

\title{
CAN CHILDREN SEE EMOTIONS IN FACES?
}

\section{Kate Lawrence $^{1 *}$, Ruth CampbelI ${ }^{2}$, and David Henry Skuse ${ }^{3}$}

${ }^{1}$ Department of Psychology, St Mary's University, Twickenham, London, UK, ${ }^{2}$ Deafness Cognition and Language Centre, University College London, London, UK, ${ }^{3}$ Behavioural and Brain Sciences Unit, UCL Great Ormond Street Institute of Child Health, London, UK

\section{REVIEWED BY:}

JEYA

16 YEARS OLD
One way in which we figure out how people are feeling is by looking at their faces. Being able to do this allows us to react in the right way in social situations. But, are young children good at recognizing facial expressions showing emotion? And how does this ability develop throughout childhood and the teenage years? Children are able to recognize certain emotions very well when they are just 6 years old, but become better at recognizing other emotions as they grow older. At all ages, girls seem to have less difficulty than boys in recognizing emotions. Hormones that our bodies produce at puberty do not only influence how our bodies develop but also influence how our brains develop and how we change emotionally. Understanding more about the typical development of emotion recognition can guide us in helping children who have difficulties with these skills.

\section{INTRODUCTION}

Have you ever thought about how you can tell what somebody else is feeling? Sometimes, friends might tell you that they are feeling happy or sad but, even if they do not tell you, I am sure that you would be able to make a good guess about what kind of mood they are in. You might get a clue from 
the tone of voice that they use. For example, they may raise their voice if they are angry or talk in a shaky way if they are scared. The other main clue you might use to tell what a friend is feeling would be to look at his or her facial expression. We have lots of muscles in our faces which enable us to move our face into lots of different positions. This happens spontaneously (without us planning for it to happen) when we feel a particular emotion. Look away from this article and try to make your face look how it would if you were feeling sad. Have you turned down your mouth and maybe looked down with your head and eyes? Isn't it amazing that the way we feel makes our faces behave in a particular way?

Scientists have discovered that, all across the world, humans tend to display the same expressions in response to various emotions. So, if you looked at an Eskimo from Canada, a school child from the USA, or a farmer from India, their facial expressions would look very similar if they were feeling sad. There are set patterns for how the facial muscles move, and therefore how the face looks, that are the same across different countries, cultures, and ethnicities. This was demonstrated over 40 years ago by scientists Ekman and Friesen [1] and has been confirmed many times since. All across the world, the emotions of happiness, sadness, anger, fear, disgust, and surprise are shown on our faces in similar ways (see Figure 1). The ability to recognize how somebody might be feeling is a very important skill in life, as it enables us to respond appropriately in social situations with our family, friends, teachers, and even strangers.

Do you think we are born able to recognize facial expressions or that this is a skill we learn as we grow, in a similar way to how we might learn to talk or read or write? Well, to some extent, the answer is a bit of each. Scientists have done experiments with babies as young as 7 months old to show that when they look at two faces with different facial expressions, they are able to tell the difference between the two expressions. Some of you may be thinking, "how do the scientists know that the babies can tell the difference, if they can't talk?" Good question! One of the ways they do this is to measure the time that babies look at different types of facial expressions. Babies will look at certain facial expressions (for example, fear) longer than others.

\section{FIGURE}

Children from different countries, cultures, and ethnicities use the same facial expressions to show emotions.

What are these children feeling? Credit: http:// streetsill.com

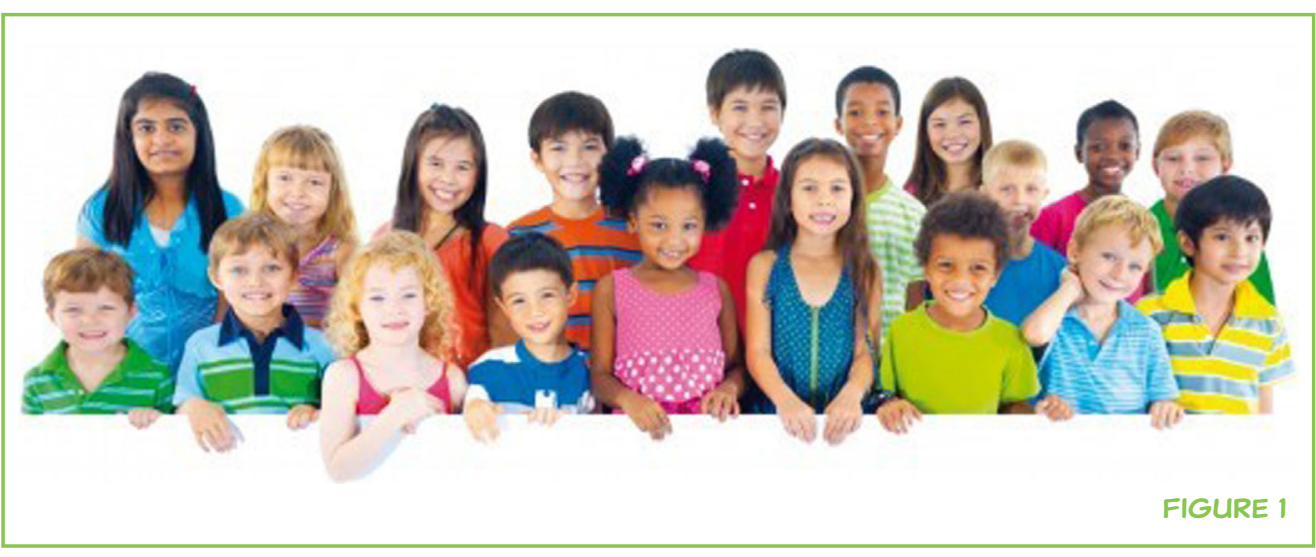


This increased amount of time spent looking tells us that the babies can see a difference between different facial expressions. However, the ability to accurately say which emotion someone is feeling based on their facial expression improves a lot between early childhood and adulthood. Although we have some ability to do this early on in life, we get much better at this skill as we grow older. The researchers Herba and Phillips [2] discuss this in their 2004 review of the topic.

Even though lots of scientific studies have been conducted looking at the ability to recognize emotions, there are no previous studies that look at emotion recognition abilities in children from 6 to 16 years using the same test with all children. Some studies have used techniques that ask children to match faces to the names of emotions. Other tests make complicated emotions by combining pictures of two different facial expressions and determine children's ability to judge which of the two emotions is shown. We felt it was important to look at a large age range using the same test on all children, because in order to understand how an ability develops, it is important that you are making fair and balanced comparisons.

Why is it important to know how good children are at recognizing facial expressions? You may think this is a rather boring topic to study. There are two main reasons this interests us. First, it is important to understand how and when this skill develops in childhood, so that we will know whether children are similar to adults in figuring out how people are feeling. If children are not as good at telling how people are feeling from their facial expressions, it might be helpful for parents or teachers to give the children extra clues. For example, maybe a teacher should not only give the class an angry look but also explain to the class that she is feeling angry because they would not stop talking. Second, not all children develop the ability to recognize emotions in the typical way, and this can have a big impact on how they are able to get along with people. We sometimes see difficulties recognizing emotions in children with behavior problems and those with autism. Furthermore, Collin and others [3] reported that children with a wide range of mental health difficulties (from attention deficit hyperactivity disorders to eating disorders) can experience difficulties recognizing emotion from facial expressions. If we are able to find out how children generally score on emotion recognition tests, then we can identify those children who have difficulties and provide them with extra help in developing these skills. In a similar way to a child who receives additional help because they have difficulty reading books, we might be able to offer help to children who have difficulty in reading faces.

\section{STUDY}

For our study, we tested 478 children from primary schools (aged 6-11 years) and secondary schools (aged 11-16 years) within the UK. Their parents gave 
permission for them to take part in the study. A computerized version of an emotion recognition test, which was designed by Ekman and Friesen [4], was developed for this study, which asked children to choose the emotion they thought people were feeling based pictures of facial expressions. Sixty pictures of faces (10 of each emotion) were shown one at a time on a computer screen (see Figure 2 for some examples). For each face, children clicked on the emotion label (happy, sad, angry, fearful, disgusted, or surprised) that best described what they thought the person in the picture was feeling. Each child got a score (out of 100) for recognizing the emotions shown in the facial expressions.

\section{RESULTS}

When we looked at the results from the tests we made some interesting discoveries. First, we found that children as young as 6 years old are able to correctly recognize facial expressions some of the time. However, as children get older they become consistently better at recognizing facial expressions (see Figure 3). Another interesting finding was that girls seemed to find this test easier than boys and judged more of the emotions correctly. This means that, on the whole, girls have an easier time knowing what people might be feeling based on their facial expressions.

Do you think it would be equally easy to recognize whether somebody is feeling sad or whether they are feeling fearful? The results of this study

\section{FIGURE 2}

Different facial expressions of emotion from the test created by Ekman and Friesen [4] and used in our study.

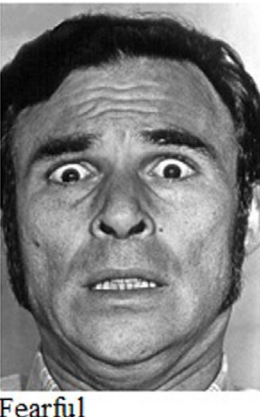

Fearful

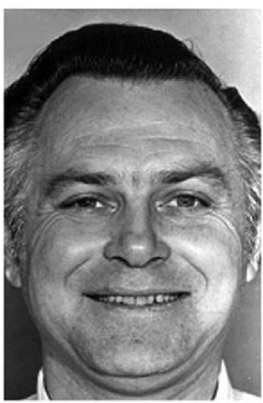

Happy

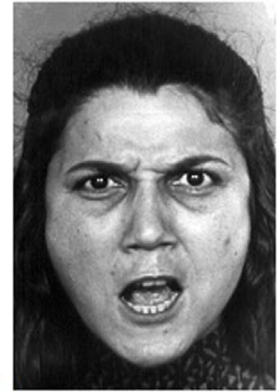

Angry

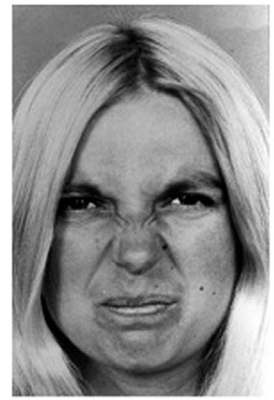

Disgusted
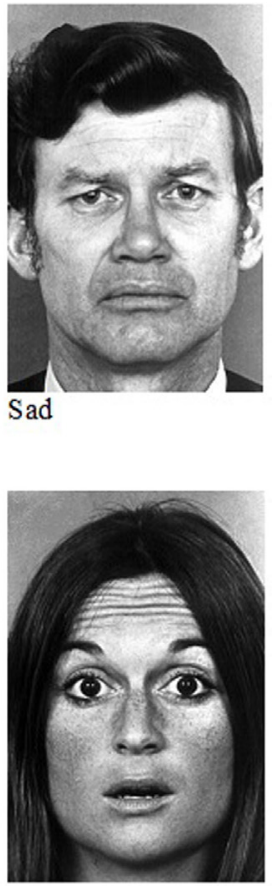

Surprised 


\section{FIGURE 3}

Line graph showing emotion recognition accuracy for boys and girls. Accuracy improves across childhood and adolescence and girls are more accurate than boys at all ages.

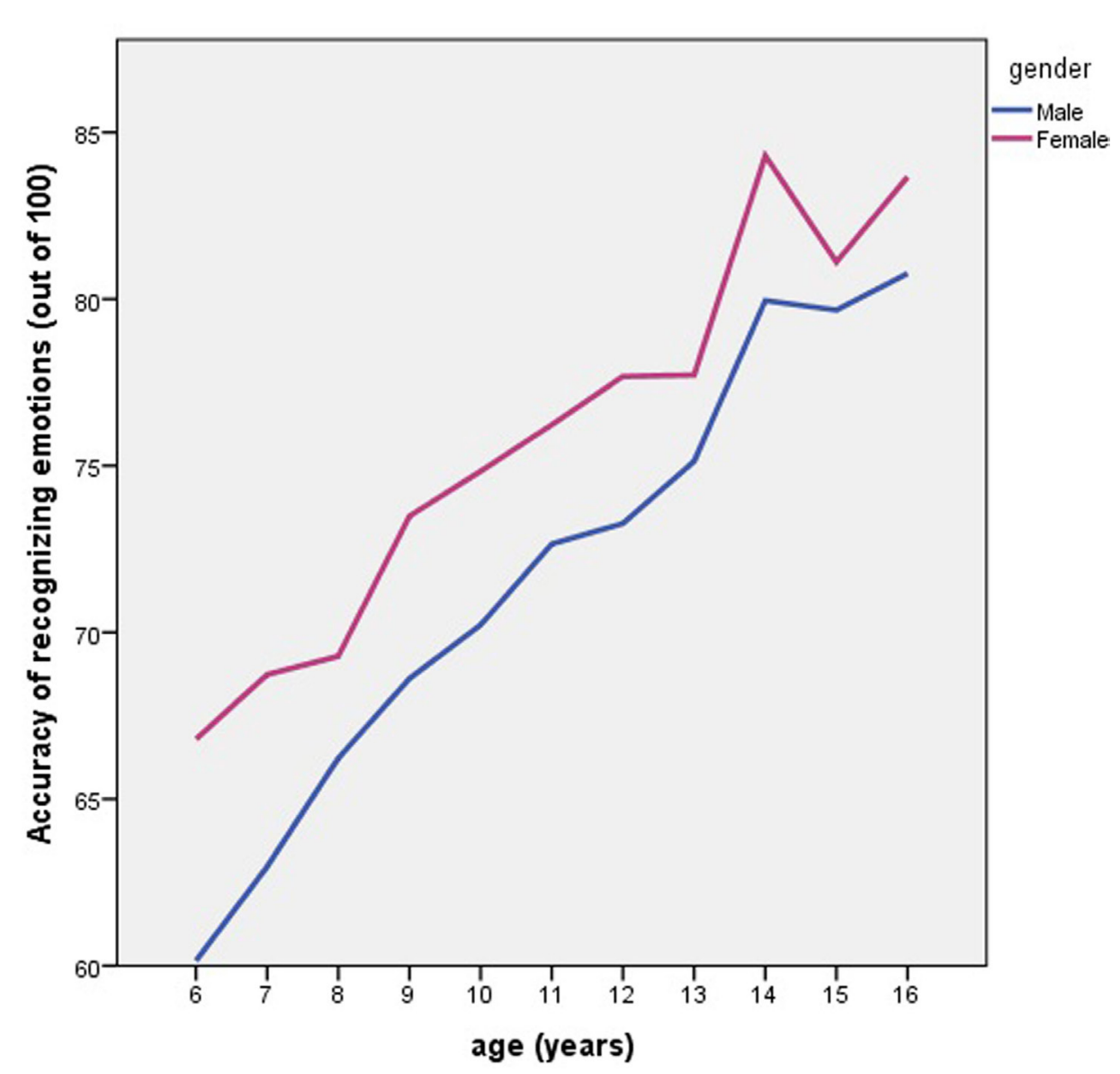

FIGURE 3

suggest it is not. Really, young children are very good at recognizing some facial expressions. The emotions of happiness, sadness, and anger were recognized almost as well by the 6 year olds as they were by the 16 year olds! This tells us that, even as a young child, you were probably quite good at telling when your mum was angry with you, just by looking at her face. I am sure you can picture the look now! However, other expressions (such as fear, disgust, and surprise) seem a bit trickier to figure out, and our ability to recognize these emotions improves throughout childhood and the teenage years (Figure 4).

One factor that may influence the improvement in recognizing emotions during the teenage years is the hormones that your body produces. As you grow from a child into an adult, your body changes in various ways and these changes are caused by hormones which are released in your body. The process is called puberty. The hormones released make you grow taller and your body become more adult-like in its appearance, but did you know that these hormones also affect how your brain develops? Scientists who study the brain [5] have found that changes in the way the brain develops during puberty are related to changes in behavior. Brain cells re-organize their connections with each other during this time and it is possible that, 


\section{FIGURE 4}

Chart showing that the ability to recognize different emotions develops at different ages. Emotion symbols are shown when children at that age can accurately recognize that emotion 7 out of 10 times. The emotions examined are listed in the key.

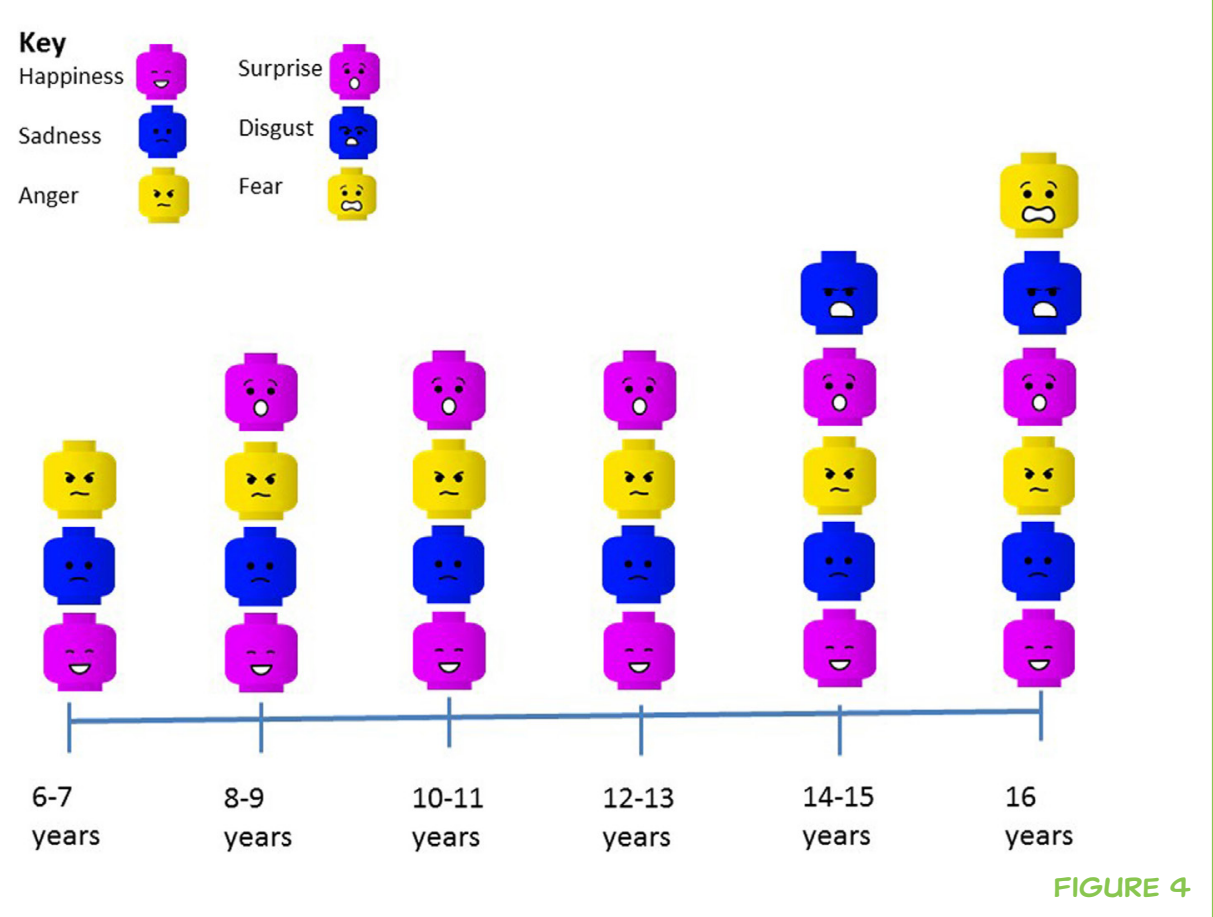

during this reorganization, our ability to recognize emotions gets a little worse before it starts to improve (see Figure 3 ). Even though young children in our study were good at recognizing angry faces, they got even better at it during the teenage years. We found that the ability of children and teenagers to recognize facial expressions of anger and disgust was related to the stage of puberty they were in. Those children who were more developed in terms of their puberty were significantly better at recognizing anger and disgust than those who were in an earlier stage of puberty. This suggests to us that the same hormones that produce the physical changes associated with puberty also cause changes in your brain that improve your ability to recognize certain emotions. Not only do the hormones make you become more adult-like physically but they also help you to become more adultlike emotionally too.

\section{CONCLUSION}

In our research, we wanted to understand the development of facial emotion recognition skills throughout childhood and the teenage years. Our study has shown us that:

- Children as young as 6 years old are good at recognizing expressions of happiness, sadness, and anger.

- We get better at recognizing other emotions as we get older.

- Our ability to recognize facial expressions of emotion improves throughout childhood and the teenage years.

- Girls seem to have less difficulty than boys at recognizing facial expressions of emotion. 
- Children improve in their ability to recognize expressions of anger and disgust as they move through puberty.

These results are interesting because they tell us more than we previously knew about how the ability to recognize facial expressions develops through childhood and the teenage years. This information could be useful for people who work with children and teenagers. The results from the study could be used to compare the emotion recognition skills of any young person, aged between 6 and 16 years, to other children their age. In this way, children who have difficulties in recognizing facial expressions could be identified in the same way that children who have difficulties with reading may be identified using reading tests.

Our ability to recognize emotions in other people has a big impact on how well we are able to get along with people in social situations. A difficulty in this skill could lead to problems in making and keeping friendships, getting along with others, and behaving appropriately in social situations. Children identified with difficulties in emotion recognition could potentially receive extra support to help develop these skills, which may help them cope better in social situations. Next time you look at someone and think you know what they are feeling just from how their face looks, think how difficult it would be if you could not use information from faces in this way. Just like having problems reading books, having difficulties reading faces can make life pretty tricky at times. The more we can find out about this skill, the more we can help people who may have problems with emotion recognition.

\section{ACKNOWLEDGMENTS}

This research was partially supported by The National Alliance for Autism Research and the Nancie Lurie Marks Family Foundation. Many thanks to Paul Ekman for providing the faces for the study and to the schools and children involved, who gave their time so enthusiastically. Finally, we are very grateful to Kate's sons, Jack and Luke, for their brilliant advice and editorial input on this article.

\section{ORIGINAL ARTICLE REFERENCE}

Lawrence, K., Campbell, R., and Skuse, D. 2015. Age, gender, and puberty influence the development of facial emotion recognition. Front. Psychol. 6:761. doi:10.3389/ fpsyg.2015.00761

\section{REFERENCES}

1. Ekman, P., and Friesen, W. V. 1971. Constants across cultures in the face and emotion. J. Pers. Soc. Psychol. 17(2):124-9. doi:10.1037/h0030377 
2. Herba, C., and Phillips, M. 2004. Annotation: development of facial expression recognition from childhood to adolescence: behavioural and neurological perspectives. J. Child Psychol. Psychiatry 45(7):1185-98. doi:10.1111/j.1469-7610.2004.00316.x

3. Collin, L., Bindra, J., Raju, M., Gillberg, C., and Minnis, H. 2013. Facial emotion recognition in child psychiatry: a systematic review. Res. Dev. Disabil. 34(5): 1505-20. doi:10.1016/j.ridd.2013.01.008

4. Ekman, P., and Friesen, W. V. 1976. Pictures of Facial Affect. Palo Alto, CA: Consulting Psychologists Press.

5. Mills, K., Goddings, A., and Blakemore, S. 2014. Drama in the teenage brain. Front. Young Minds 2:16. doi:10.3389/frym.2014.00016

SUBMITTED: 28 January 2016; ACCEPTED: 08 August 2016; PUBLISHED ONLINE: 25 August 2016.

EDITED BY: Beatriz Luna, University of Pittsburgh, USA

CITATION: Lawrence K, Campbell R and Skuse DH (2016) Can Children See Emotions in Faces? Front. Young Minds 4:15. doi:10.3389/frym.2016.00015

CONFLICT OF INTEREST STATEMENT: The authors declare that the research was conducted in the absence of any commercial or financial relationships that could be construed as a potential conflict of interest.

COPYRIGHT ( 2016 Lawrence, Campbell and Skuse. This is an open-access article distributed under the terms of the Creative Commons Attribution License (CC BY). The use, distribution and reproduction in other forums is permitted, provided the original author(s) or licensor are credited and that the original publication in this journal is cited, in accordance with accepted academic practice. No use, distribution or reproduction is permitted which does not comply with these terms.

\section{REVIEWED BY}

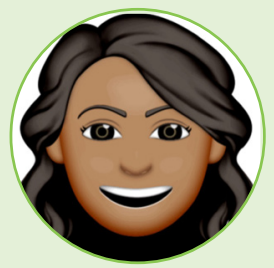

\section{JEYA, 16 YEARS OLD}

I am Jeya, and I love science. Science is my favorite subject because it answers many questions about natural phenomena. I also enjoy music, I have played the flute for 6 years at my school band, and I plan on continuing it. Playing an instrument is fun and challenging.

\section{AUTHORS}

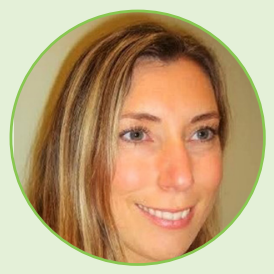

\section{KATE LAWRENCE}

I am a psychologist who is interested in the development of social skills throughout childhood and adolescence. I study these skills in young people with disorders, such as autism, and also in schoolchildren without any psychological problems. I am keen to use this research to help develop ways to improve the lives of children who have difficulties with social skills and emotion recognition. Outside of work, I enjoy playing sport with my husband and two boys and family holidays in Cornwall. *kate.lawrence@stmarys.ac.uk 


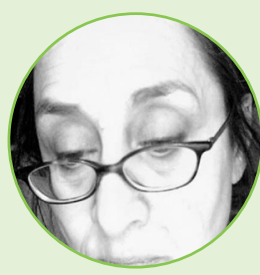

\section{RUTH CAMPBELL}

I worked in psychology and brain research for more than 30 years. I am interested in how people perceive faces, and also in how different skills (such as reading and spelling) develop in childhood. Some people can show very different ways of "reading faces," and I explore why this could happen and whether it tells us something interesting about how the brain works. I like playing Dobble with grandson Hal (aged 7 years) and going shopping with Dot (granddaughter, aged 12 years). I like singing in a choir, but am not very good.

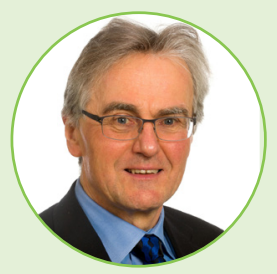

\section{DAVID HENRY SKUSE}

I am a child psychiatrist who is interested in the problems of boys and girls who do not develop good social skills during their childhood and adolescence. I see many young people with problems in what we call social communication, some of whom have Asperger syndrome. We became interested in whether typically developing children also vary widely in their ability to recognize other people's feelings from their expressions, as well as in other social skills. Our research has shown that many of us do indeed have some problems in social communication, but most people are not much affected in their everyday life, because they find ways of compensating or disguising those difficulties. Outside of work, I enjoy traveling, cycling, and spending time with our family and their own children. 